\title{
Hexadecatrienoic Acid n-3
}

National Cancer Institute

\section{Source}

National Cancer Institute. Hexadecatrienoic Acid n-3. NCI Thesaurus. Code C68379.

A polyunsaturated long-chain fatty acid with a 16-carbon backbone and 3 double bonds

originating from the 3rd, 6th and 9th positions from the methyl end. 